\title{
QUASIHARMONIC CLASSIFICATION OF RIEMANNIAN MANIFOLDS ${ }^{1}$
}

\author{
MITSURU NAKAI AND LEO SARIO
}

\begin{abstract}
In the study of the structure of the space of biharmonic functions it is often necessary to impose some nondegeneracy condition on the base manifold with respect to quasiharmonic functions (cf. [2], [4]). For this reason it is useful to introduce various quasiharmonically degenerate classes of Riemannian manifolds and to investigate relations among them. This is the purpose of the present note.
\end{abstract}

1. Quasiharmonic degeneracy. Let $R$ be a noncompact orientable $C^{\infty}$-manifold of dimension $m \geqq 2$ with $C^{\infty}$ Riemannian metric $d s^{2}=$ $g_{i j} d x^{i} d x^{j}$. The corresponding Laplace-Beltrami operator is

$$
\Delta \cdot=-g^{-1 / 2} \sum_{i=1}^{m} \frac{\partial}{\partial x^{i}}\left(\sum_{j=1}^{m} g^{1 / 2} g^{i j} \frac{\partial \cdot}{\partial x^{j}}\right)
$$

where $g=\operatorname{det}\left(g_{i j}\right)$ and $\left(g^{i j}\right)=\left(g_{i j}\right)^{-1}$. We call a function $u$ quasiharmonic if $\Delta u=$ const $\neq 0$, and denote by $Q=Q(R)$ the class of quasiharmonic functions $u$ on $R$ normalized by $\Delta u=1$. Such functions are superharmonic on $R$.

Following [5], we denote the classes of nonnegative, bounded, and Dirichlet finite functions by $P, B$, and $D$ respectively, and we set $B D=$ $B \cap D$. Similarly we write $Q X(X=P, B, D$, or $B D)$ for $Q \cap X$. We are interested in the question as to when $Q X=\varnothing$.

2. Characterization of null classes. We denote by $O_{Q X}$ the class of Riemannian manifolds $R$ on which $Q X(R)=\varnothing$, and by $O_{G}$ the class of parabolic manifolds.

Let $G(x, y)=G_{R}(x, y)$ be the harmonic Green's function on $R \notin O_{G}$, and set $G(x, y)=\infty$ on $R \in O_{G}$. Denote by $d y$ the Riemannian volume element $(g(y))^{1 / 2} d y^{1} \cdots d y^{m}$.

Received by the editors September 11, 1970.

AMS 1970 subject classifications. Primary 31B30.

${ }^{1}$ The work was sponsored by the U.S. Army Research Office-Durham, Grant DA-ARO-D-31-124-70-G7, University of California, Los Angeles.

(c) American Mathematical Society 1972 
THEOREM 1. The classes $O_{Q X}$ are characterized in terms of $G(x, y)$ as follows:

(2)

$$
\begin{aligned}
& R \in O_{Q P} \quad \text { if and only if } \quad \int_{R} G(x, y) d y=\infty \\
& R \in O_{Q B} \quad \text { if and only if } a=\sup _{x \in R} \int_{R} G(x, y) d y=\infty \\
& R \in O_{Q D} \text { if and only if } b=\int_{R \times R} G(x, y) d x d y=\infty \\
& R \in O_{Q B D} \quad \text { if and only if } a=\infty \text { or } \quad b=\infty .
\end{aligned}
$$

3. Proof. Let $u \in Q(R)$ and take a regular subregion $\Omega$ of $R$ containing a given point $x$. Denote by $H_{u}^{\Omega}$ the harmonic solution of the Dirichlet problem on $\Omega$ with boundary values $u$. By Stokes' formula,

$$
\begin{gathered}
\int_{\Omega-B}\left[\left(u(y)-H_{u}^{\Omega}(y)\right) \Delta_{y} G_{\Omega}(x, y)-G_{\Omega}(x, y) \Delta_{y}\left(u(y)-H_{u}^{\Omega}(y)\right)\right] d y \\
=-\int_{\partial \Omega-\partial B}\left[\left(u(y)-H_{u}^{\Omega}(y)\right) * d_{y} G_{\Omega}(x, y)\right. \\
\left.-G_{\Omega}(x, y) * d_{y}\left(u(y)-H_{u}^{\Omega}(y)\right)\right],
\end{gathered}
$$

with $B$ a small geodesic ball about $x$ of radius $\varepsilon$. On letting $\varepsilon \rightarrow 0$, we obtain the Riesz representation

$$
u(x)=H_{u}^{\Omega}(x)+\int_{\Omega} G_{\Omega}(x, y) d y
$$

on $\Omega$ (cf., e.g., [1]). Again by Stokes' formula,

$$
\begin{aligned}
D_{\Omega}\left(\int_{\Omega} G_{\Omega}(\cdot, y) d y\right) & =\int_{\Omega}\left(\int_{\Omega} G_{\Omega}(x, y) d y \cdot \Delta_{x} \int_{\Omega} G_{\Omega}(x, y) d y\right) d x \\
& =\int_{\Omega \times \Omega} G_{\Omega}(x, y) d x d y .
\end{aligned}
$$

We conclude that

$$
D_{\Omega}(u)=D_{\Omega}\left(H_{u}^{\Omega}\right)+\int_{\Omega \times \Omega} G_{\Omega}(x, y) d x d y .
$$

If $\int_{R} G(x, y) d y$ exists, then it is of class $C^{2}$, and

$$
\Delta_{x} \int_{R} G(x, y) d y=1
$$

(cf., e.g., [3]). On letting $\Omega \rightarrow R$ in (5), we obtain

$$
D_{R}\left(\int_{R} G(\cdot, y) d y\right)=\int_{R \times R} G(x, y) d x d y \leqq \infty .
$$


Suppose there exists a $u \in Q P$. Since $u$ is positive and superharmonic on $R, h(x)=\lim _{\Omega \rightarrow R} H_{u}^{\Omega}(x)$ exists and a fortiori

$$
\int_{R} G(x, y) \dot{d} y=\lim _{\Omega \rightarrow R} \int_{\Omega} G_{\Omega}(x, y) d y=u(x)-h(x)<\infty .
$$

If $u \in Q B$, then since $\left|H_{u}^{\Omega}(x)\right| \leqq \sup _{\partial \Omega}|u|$ for every $x \in \Omega$, (4) implies

$$
\int_{\Omega} G_{\Omega}(\cdot, y) d y \leqq 2 \sup _{R}|u|
$$

and consequently $a<\infty$. If $u \in Q D$, then (6) and (7) give $b<\infty$. A fortiori, if $u \in Q B D$, then $a<\infty$ and $b<\infty$.

Conversely let $v(x)=\int_{R} G(x, y) d y$. If it is finite or bounded on $R$, then $v \in Q P$ or $Q B$. If $b<\infty$, then $v(x)$ is finite and (7) implies $v \in Q D$. Consequently, if $a<\infty$ and $b<\infty$, then $v \in Q B D$.

4. Strict inclusion relations. By means of the characterizations in Theorem 1 we shall prove:

THEOREM 2. The following strict inclusion relations are valid:

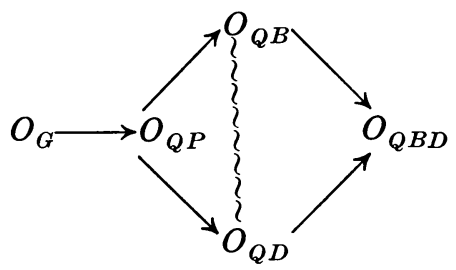

Here $X \rightarrow Y$ stands for $X \subsetneq Y$, and $X \sim \sim \sim Y$ means $X \notin Y$ and $Y \ddagger X$.

5. An auxiliary metric. The inclusion $O_{G} \subset O_{Q P} \subset O_{Q B}, O_{Q P} \subset$ $O_{Q D}$, and $O_{Q B} \cup O_{Q D} \subset O_{Q B D}$ follow immediately from (2). The simplest example showing the strictness of the inclusion $O_{G} \subsetneq O_{Q P}$ is the Euclidean space $E^{m}(m \geqq 3)$. To construct examples showing the strictness of the other inclusion relations, we make the following preliminary observation.

Take a hyperbolic region $S$ in the $z$-plane. Denote by $\Delta_{e}$ the Laplacian $-4 \partial^{2} / \partial z \partial \bar{z}$ and by $G(z, \zeta)$ the Green's function on $S$. Let $v$ be a $C^{\infty}$ superharmonic function on $S$ with $\Delta_{e} v>0$ and suppose that $v$ is a potential, i.e., the greatest harmonic minorant of $v$ is zero. Let $\lambda(z)=\Delta_{e} v(z)$. Consider the Riemannian manifold $R$ with base manifold $S$ and metric $(\lambda(z))^{1 / 2}|d z|$. The Laplace-Beltrami operator $\Delta$ is simply $\Delta=\lambda^{-1} \Delta_{e}$. Thus a function is harmonic on $R$ if and only if it is so on $S$, i.e., $H(R)=$ $H(S)$. The volume element $d V$ on $R$ is given by $d V(z)=\frac{1}{2} i \lambda(z) d z \wedge d \bar{z}$. 
In view of $v \in C^{\infty}$ we obtain on replacing $u$ by $v$ in (3) and letting $\varepsilon \rightarrow 0$,

$$
v(z)=H_{v}^{\Omega}(z)+\frac{i}{2} \int_{\Omega} G_{\Omega}(z, \zeta) \Delta_{e} v(\zeta) d \zeta \wedge d \zeta .
$$

Since $v$ is a potential, we have $\lim _{\Omega \rightarrow R} H_{v}^{\Omega}(z)=0$. Therefore

and

$$
\int_{R} G(z, \zeta) d V(\zeta)=v(z)
$$

$$
\int_{R \times R} G(z, \zeta) d V(z) d V(\zeta)=\frac{i}{2} \int_{R} v(z) \Delta_{e} v(z) d z \wedge d \bar{z} .
$$

6. Example 1. Let $R$ be as in $\S 5$, and choose

$$
S=\{0<|z|<1\}, \quad v(z)=\log (1-\log |z|) .
$$

Then

$$
\Delta_{e} v(z)=|z|^{-2}(1-\log |z|)^{-2}>0
$$

and, for $\Omega(\rho)=\{\rho<|z|<1\}$,

$$
H_{v}^{\Omega(\rho)}(z)=\frac{\log (1-\log \rho) \cdot \log |z|}{\log \rho} .
$$

Since this tends to zero as $\rho \rightarrow 0, R$ qualifies as a manifold of $\S 5$. By (9) we have

$$
\int_{R} G(z, \zeta) d V(\zeta)=\log (1-\log |z|),
$$

which is finite but not bounded on $R$. This with (2) implies that $R \notin O_{Q P}$ and $R \in O_{Q B} \subset O_{Q B D}$.

Similarly (10) gives

$$
\begin{aligned}
\int_{R \times R} G(z, \zeta) d V(z) & d V(\zeta) \\
= & \frac{i}{2} \int_{R \times R} \log (1-\log |z|) \cdot|z|^{-2}(\log |z|)^{-2} d z \wedge d \bar{z}<\infty .
\end{aligned}
$$

Again by (2) we conclude that $R \notin O_{Q D}$. The example (11) thus serves to show that $O_{Q P} \subsetneq O_{Q B}, O_{Q D} \subsetneq O_{Q B D}$, and $O_{Q B} \notin O_{Q D}$.

7. Example 2. Let $R$ be again as in $\S 5$ but now choose

$$
S=\{0<|z|<1\}, \quad v(z)=(1-|z|)^{1 / 2} .
$$


Then

$$
\Delta_{e} v(z)=4^{-1}(2-|z|)|z|^{-1}(1-|z|)^{-1 / 2-1}>0 .
$$

Since $v$ vanishes on $|z|=1$ and is bounded on $R$, it is clearly a potential on $R$. Therefore $R$ satisfies the condition of $\S 5$. By (9), we have

$$
\int_{R} G(z, \zeta) d V(\zeta)=(1-|z|)^{1 / 2}
$$

In view of (2) we conclude that $R \notin O_{Q B}$ and a fortiori $R \notin O_{Q P}$. Moreover it follows from (10) that

$\int_{R \times R} G(z, \zeta) d V(z) d V(\zeta)=\frac{i}{2} \int_{R \times R}(1-|z|)^{1 / 2} \cdot \Delta_{e}(1-|z|)^{1 / 2} d z \wedge d \bar{z}=\infty$.

This with (2) gives $R \in O_{Q D} \subset O_{Q B D}$. The example (14) thus yields the relations $O_{Q P} \varsubsetneqq O_{Q D}, O_{Q B} \varsubsetneqq O_{Q B D}$, and $O_{Q D} \notin O_{Q B}$.

The proof of Theorem 2 is herewith complete.

\section{REFERENCES}

1. C. Constantinescu and A. Cornea, Ideale Ränder Riemannscher Flächen, Ergebnisse der Mathematik und ihrer Grenzgebiete, Heft 32, Springer-Verlag, Berlin, 1963. MR 28 \#3151.

2. Y. K. Kwon, L. Sario and B. Walsh, Behavior of biharmonic functions on Wiener's and Royden's compactifications, Ann. Inst. Fourier (Grenoble) (to appear).

3. C. Miranda, Equazioni alle derivate parziali di tipo ellittico, Ergebnisse der Mathematik und ihrer Grenzgebiete, Heft 2, Springer-Verlag, Berlin, 1955; English transl., Springer-Verlag, Berlin, 1970. MR 19, 421.

4. M. Nakai and L. Sario, Biharmonic classification of Riemannian manifolds, Bull. Amer. Math. Soc. 77 (1971), 432-436.

5. L. Sario and M. Nakai, Classification theory of Riemann surfaces, Die Grundlehren der math. Wissenschaften, Band 164, Springer-Verlag, Berlin and New York, 1970.

Department of Mathematics, University of California, los Angeles, CaliFORNIA 90024

Current address: 52 Eguchi, Hinaga, Chita, Aichi 478, Japan

Current address: 521 Georgina Avenue, Santa Monica, California 90402 